[Review]

doi: 10.3866/PKU.WHXB201112222

www.whxb.pku.edu.cn

\title{
铱配合物在磷光化学传感器中的应用
}

郭远辉 梅群波 ${ }^{*}$ 颜 芳 王玲霞 翁洁娜张 涁 黄 维*

(有机电子与信息显示国家重点实验室培育基地, 南京邮电大学信息材料与纳米技术研究院, 南京 210046)

摘要: 由于在生物领域和物联网领域的广泛应用, 化学传感器在近期发展迅速. 相比于纯有机分子的荧光化 学传感器, 基于重金属配合物的磷光化学传感器由于发光寿命长, 斯托克斯位移大等优点越来越引起人们的广 泛关注. 重金属铱配合物三线态寿命短, 发光效率高而且配合物的发射波长容易受配体的改变而发生变化, 因 此成为最好的磷光传感器材料之一. 本文介绍了铱配合物在磷光化学传感器领域中的应用, 具体包括: 阳离子 传感器、阴离子传感器、氧分子传感器、氨基酸传感器、 $\mathrm{pH}$ 传感器等, 并指出了相比于其它磷光化学传感器, 基 于铱配合物的磷光化学传感器的优势以及目前所存在的问题, 最后, 对基于铱配合物的磷光化学传感器的研究 和发展方向进行了展望.

关键词：化学传感器; 离子型铱配合物; 中性铱配合物; 磷光; 能量传递 中图分类号: 0644

\section{Application of Iridium(III) Complexes in Phosphorescent Chemosensors}

\author{
GUO Yuan-Hui MEI Qun-Bo* YAN Fang WANG Ling-Xia \\ WENG Jie-Na ZHANG Bin HUANG Wei* \\ (Key Laboratory for Organic Electronics \& Information Displays, and Institute of Advanced Materials, Nanjing \\ University of Posts \& Telecommunications, Nanjing 210046, P. R. China)
}

\begin{abstract}
Chemosensors have developed quickly because they are widely used in biology. Compared to organic fluorescent chemosensors, phosphorescent chemosensors based on heavy metal complexes have attracted great attention because of distinctive merits such as relatively long lifetimes and significant Stokes shifts. Iridium complexes had been successfully used as phosphorescent chemosensors because of their relatively short excited state lifetime, high photoluminescence efficiency, and wide range emission colors that can be tuned by the coordinated ligands. In this review, we have summarized the applications of iridium complexes in cation, anion, oxygen, amino acid, and pH sensors. Furthermore, the advantages and disadvantages of these chemosensors have been compared to others. Finally, some prospects for future study are proposed.
\end{abstract}

Key Words: Chemosensor; Charged iridium complex; Neutral iridium complex; Phosphorescence; Energy transfer

\section{1 引言}

化学传感器是指对各种化学物质敏感并能将
其浓度和种类转换为电信号进行检测的仪器, 其必 须具有对待测化学物质的分子结构或形态选择性

Received: October 10, 2011; Revised: December 14, 2011; Published on Web: December 22, 2011.

"Corresponding authors. MEI Qun-Bo, Email: iamqbmei@njupt.edu.cn. HUANG Wei, Email: iamwhuang@njupt.edu.cn; Tel: +86-25-85866396. The project was supported by the National Key Basic Research Program of China (973) (2009CB930601), National Natural Science Foundation of China (50803027, 50903001, 20905038), and Natural Science Fund for Colleges and Universities in Jiangsu Province, China (08KJD430020). 国家重点基础研究发展规划项目(973) (2009CB930601), 国家自然科学基金(50803027, 50903001, 20905038)及江苏省高校自然科学基础研究 面上项目(08KJD430020)资助

(C) Editorial office of Acta Physico-Chimica Sinica 
俘获的功能( (接受器功能)和将俘获的化学变量有效 转换为电信号输出的功能(转换器功能). 这些传感 器体积小、携带方便、费用低, 而且可以在苛刻的条 件下使用. 当不同量的化学物质加入到体系后, 可 以得到放大了很多倍的输出信号, 根据输出信号推 断出化学物质的种类和浓度. 研究者根据某些化学 反应的反应条件和某些功能团在待检测物质存在 下被功能化而制得了各类化学传感器. ${ }^{1,2}$ 按照被检 测物质分类, 可以分为: 气体传感器、湿度传感器、 离子传感器和生物传感器等, 传感器接收这些信号 可以将其转换为其它信号输出, 这些信号包括: 荧 光发射、紫外吸收、体系颜色、电化学等. ${ }^{3-6}$ 化学传感 器按检测的信号可以分为: 光学传感器、电化学传 感器、温度敏感化学传感器等.

光敏感材料与待检测物质接触后, 它们之间的 化学反应会引起光信号的变化, 能感知这类光信号 变化的传感器称为光学传感器. 由于输出的光信号 易于辨别, 光学传感器已经被成功应用于对不同气 体、氨基酸、离子的传感. 按照发光时的能级跃迁方 式, 可分为基于纯有机小分子的荧光化学传感器和 基于重金属配合物的磷光化学传感器, 相比于荧光 化学传感器, 基于重金属配合物(如锇 $(\mathrm{Os})$ 、铱(Ir)、铂 $(\mathrm{Pt}) 、$ 钉 $(\mathrm{Ru})$ 等)磷光化学传感器由于发光寿命长, 斯 托克斯位移大, 而且不容易受背景的干扰, 灵敏度 高, 越来越引起人们的关注..$^{7-9}$ 人们可以通过改变配 合物中配体的结构和中心重金属离子的种类来改 变配合物的光物理和电化学性质, 使得设计这些传 感器更加简单. 磷光重金属配合物的激发态性质非 常复杂, 通常将对某些待检测物质具有特异性识别 能力的功能团引入到配体结构中而制得性能良好 的化学传感器.

相比于其他重金属配合物, 铱配合物由于其三 线态寿命短, 有较好的磷光性能, 成为研究最多的 重金属配合物, 这是由于铱的原子序数较大, 可使 配合物产生很强的自旋轨道耦合, 有利于磷光发 射, 而且铱金属离子中的 $d$ 轨道分裂较大, 避免与配 合物的金属与配体之间的能量传递(MLCT)态相互 作用而降低磷光发射效率. ${ }^{10,11}$ 相比于 Ru、Os 等配合 物的激发态仅限制在 MLCT 态, ${ }^{12-15}$ 铱配合物的激发 态包括 MLCT 态, 配体与配体之间的能量传递 (LLCT)态和配体内部的能量传递(ILCT)态等, ${ }^{16-18}$ 使 得重金属铱配合物的发射波长、强度以及电化学性 能会随着环境和功能团的改变而发生的变化更加
明显, 这些性能使得重金属铱配合物被广泛使用于 制备多响应的磷光化学传感器. 下面我们主要介绍 基于重金属铱配合物的各种不同的传感器.

\section{2 基于重金属铱配合物的传感器}

\section{1 阳离子传感器}

金属阳离子在生活和环境中的地位非常明 显, ${ }^{19-21}$ 金属元素是人体组成的必需元素, 某些金属 离子在细胞分裂过程中起促进调节的作用, 比如 $\mathrm{K}^{+} 、 \mathrm{Na}^{+} 、 \mathrm{Mg}^{2+}$ 等离子在日常生活中对神经传递和肌 肉收缩等活动起着非常重要的作用. 而一些离子对 环境和生物体存在危害性, 如 $\mathrm{Hg}^{2+} 、 \mathrm{~Pb}^{2+}$ 等, 因此越 来越多的人开始关注对这些阳离子的检测方法(如 图 1 所示). ${ }^{22,23}$

Ho 等 ${ }^{24}$ 合成了铱配合物 1 , 借助辅助配体上的 氮原子与 $\mathrm{Pb}^{2+}$ 的络合作用, 使得络合 $\mathrm{Pb}^{2+}$ 后配合物的 发光发生红移而且强度降低, 这是由于 $\mathrm{Pb}^{2+}$ 离子的 加入使得配体与配合物中心铱之间能量转移增加.

二吡啶甲基胺及其衍生物通常作为检测基团 用于阳离子传感器中, 研究者通过改变发光基团以 及发光基团与检测基团的共轭程度, 已经将这类材 料广泛使用到 $\mathrm{Zn}^{2+} 、 \mathrm{Cd}^{2+}$ 等离子的检测中. ${ }^{25-27}$ Araya 等 ${ }^{28}$ 在铱配合物的辅助配体上引入二吡啶甲基胺作 为阳离子的检测基团, 在配合物 $\mathbf{2}$ 的乙腈溶液中加 入 $\mathrm{Ni}^{2+} 、 \mathrm{Zn}^{2+} 、 \mathrm{Cd}^{2+}$ 后, 溶液的发光发生了不同的变 化, 当加入 $\mathrm{Ni}^{2+}$ 后, 溶液的光完全淬灭; 当加入 $\mathrm{Cd}^{2+}$ 后, 溶液原来的发光完全淬灭, 但是有微弱的蓝移 现象; 然而, 当 $\mathrm{Zn}^{2+}$ 加入后, 溶液的发光强度没有变 化, 最大发射波长由原来的 $640 \mathrm{~nm}$ 蓝移到 $610 \mathrm{~nm}$, 这是由于二吡啶甲基胺上的氮原子与金属络合后, 降低了其对铱配合物的给电子能力, 进而降低了配 合物的 HOMO 能级, 从而使配合物的发光蓝移. Zhao 等 ${ }^{29}$ 合成了辅助配体含有二吡啶甲基胺衍生物 的铱配合物 3-6, 不同官能团修饰的铱配合物对离 子有不同的响应, 往配合物 3 中加入 $\mathrm{Zn}^{2+}$, 配合物的 发射增强, 配合物 4 不仅对 $\mathrm{Zn}^{2+}$ 有响应, 而且对 $\mathrm{Hg}^{2+}$ 也有响应, 往配合物 $\mathbf{5}$ 中加入 $\mathrm{Cu}^{2+}$ 后, 苂光先增强然 后淬灭, 当往配合物 6 中加入 $\mathrm{Cu}^{2+}$ 后配合物的发光 只是增强. 这是由于金属阳离子与铱配合物键合 后, 配合物的激发态发生改变.

冠醚或者冠杂醚能够与阳离子很好地络合, 将 这类冠醚或者杂冠醚引入到重金属配合物中, 并且 通过改变冠醚环的大小, 或者冠杂醚上杂原子的类 


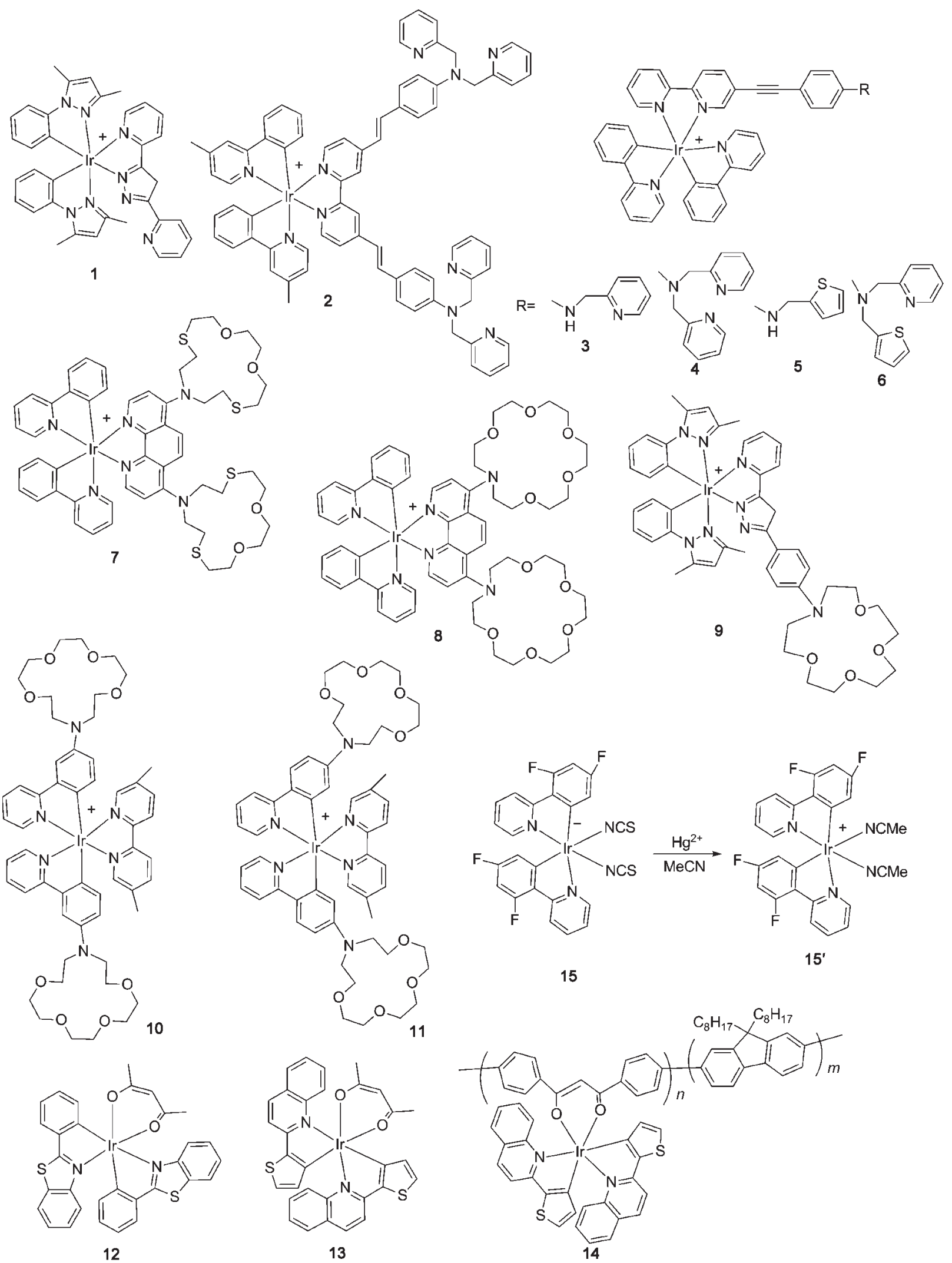

图 1 铱配合物用于阳离子传感器

Fig.1 Application of iridium complexes in chemosensors for cations 
型, 得到了一系列基于重金属配合物的阳离子磷光 传感器, ${ }^{30-32}$ 当阳离子加入后, 冠醚只与溶剂中的阳 离子络合, 排除了阴离子的干扰, 金属阳离子的加 入会改变配合物金属中心与配体的电子云分布, 进 而来改变配合物的光物理性质. 配合物 $7^{33} 、 8^{34}$ 都是 辅助配体含有冠醚或者杂冠醚的离子型铱配合物. 由于冠醚的结构不同, 配合物 $7 、 8$ 能检测的离子类 型也不同, 当 $\mathrm{Ba}^{2+}$ 加入后配合物 7 的发光显著增强, 当 $\mathrm{Ag}^{+}$加入后配合物 8 的发光也是显著增强, 这是由 于当辅助配体上的冠醚或者杂冠醚与阳离子结合 后, 辅助配体上的氮原子的供电子能力下降. 往配 合物 $9^{35}$ 中加入 $\mathrm{Ca}^{2+}$ 后, 体系的发光明显蓝移且增 强, 通过理论计算发现, 这是由于激发态从 ILCT 态 转化为 LLCT 态. 铱配合物 10 和 11 是在主配体上含 有冠醚基团, 与相同冠醚取代在辅助配体的配合物 9 相比, 检测效果完全不同, 配合物 10 和 11 本身没 有发光现象, 这是由于冠醚中的氮原子到铱中心的 分子内的电荷转移导致发光淬灭, 当往配合物 10 和 $11^{36}$ 中加入 $\mathrm{Mg}^{2+}$ 后, 配合物 10 发光增强, 而配合物 11 的发光基本没有影响, 这是由于配合物 10 与 $\mathrm{Mg}^{2+}$ 络合后，抑制了分子内氮原子到铱中心的电荷转 移, 而配合物 11 存在空间位阻效应, 不能与 $\mathrm{Mg}^{2+}$ 络合.

$\mathrm{Hg}^{2+}$ 作为软酸能够与作为软碱的硫原子作用, 将硫原子引入到铱配合物, 利用硫原子与 $\mathrm{Hg}^{2+}$ 的相 互作用可以改变配合物的光物理性能和电化学性 能, 这样就能够实现对 $\mathrm{Hg}^{2+}$ 的高灵敏度、高选择性检 测. 铱配合物 $12^{37}$ 的主配体中含有硫原子, 加入采离 子后, 配合物的发射明显地蓝移, 溶液颜色也由橙 色变为黄绿色, 铱配合物 $13^{38}$ 的发射却只是出现单 纯的减弱, 溶液发射由红色变为黄色, 通过理论计 算发现, 铱配合物 13 上的硫原子的电正性比配合物 12 的强, 当配合物与采离子反应后, 电荷分布明显 不同, 使得两者对录离子传感现象不同. 基于配合 物 12 和 13 对录离子的响应现象, 共轭聚合物 14 实 现了对 $\mathrm{Hg}^{2+}$ 的比率法检测, ${ }^{39}$ 这是由于录离子的加入 导致配合物受体磷光淬灭, 改变了聚芴主体到配合 物客体的能量转移. Yang 等 ${ }^{40}$ 对检测的机理进行了 更进一步的探讨, 根据软硬酸碱理论, $\mathrm{Hg}^{2+}$ 加入到配 合物 15 中后, 配合物被解开, 通过质谱分析发现, 形 成的空轨道与乙腈配位, 生成配合物 $\mathbf{1 5}^{\prime}$, 配合物的 发光很弱, 从而得到对 $\mathrm{Hg}^{2+}$ 的 “turn-off”型传感器.

基于铱配合物阳离子传感器是研究最早且研
究最成熟的磷光传感器, 目前已经成功实现对多种 离子的高灵敏度和高选择性的检测, 铱配合物拥有 大的斯托克斯位移, 使得检测时可以容易分辨激发 和发射, 而且铱配合物拥有长的发光寿命, 可以使 用时间分辨技术与背景苂光信号相区分来提高检 测的性噪比. 但是, 这类传感器还是有需要改进的 地方, 比如当将这类传感器应用到水体检测的时候 需要这类传感器有良好的水溶性, 但是有关这类传 感器的报告还是比较少, 因此在未来的研究中, 设 计和实现一些水溶性阳离子磷光传感器尤为重要.

\section{2 阴离子传感器}

相比于金属阳离子, 阴离子在生物体和环境中 也相当地重要, 比如 $\mathrm{F}^{-}$在牙科和治疗骨质疏松症中 的广泛应用, $\mathrm{CN}^{-}$在采矿和电镀中的使用及其对生 物体和环境的毒害作用, $\mathrm{Cl}^{-}$的含量等对生物体的某 些生理功能也有影响, 对这些阴离子的检测越来越 引起人们的广泛关注(如图 2 所示). ${ }^{41-43}$

目前对 $\mathrm{F}^{-}$的检测大致可以分为两种, 第一种是 利用氢键作用, $\mathrm{F}^{-}$是弱酸根, 可以与配合物中裸露的 氢质子结合, 进而改变配合物的光学性能, 但是 $\mathrm{CH}_{3} \mathrm{COO}^{-} 、 \mathrm{H}_{2} \mathrm{PO}_{4}^{-}$等也是弱酸根, 这使得制的 $\mathrm{F}^{-}$传感 器的选择性不高, 而且这类传感器的性能受体系酸 碱度的影响很大. Zhao 等 ${ }^{44}$ 合成离子型铱配合物 16-18, $\mathrm{F}^{-} 、 \mathrm{H}_{2} \mathrm{PO}_{4}^{-} 、 \mathrm{CH}_{3} \mathrm{COO}^{-}$的加入会使配合物的光 学性能发生改变, 研究者通过对比加入 $F^{-}$后配合物 的光谱与辅助配体上氢被乙基取代后的光谱, 证明 了引起配合物光谱变化的原因是辅助配体上的氢 被质子化. Lo 等 ${ }^{45}$ 合成了一系列辅助配体上含有取 代硫脲铱配合物 19-21, $\mathrm{F}^{-} 、 \mathrm{CH}_{3} \mathrm{COO}^{-} 、 \mathrm{H}_{2} \mathrm{PO}_{4}^{-}$的加入 使得溶液的发射强度降低, 相比于 $\mathrm{F}^{-} 、 \mathrm{H}_{2} \mathrm{PO}_{4}^{-}$, 这类 酰胺基团对 $\mathrm{CH}_{3} \mathrm{COO}^{-}$的络合能力最好. 第二种是利 用路易斯酸碱相互作用, 例如 $\mathrm{F}^{-}$对嗍原子有强的亲 和力, You 等 ${ }^{46}$ 合成了配合物 $\mathbf{2 2}$, 当加入 $\mathrm{F}^{-}$后, 配合 物的发射光谱发生明显的变化, 先是逐渐淬灭, 后 来又逐渐增强, 并且发生明显的红移, 溶液颜色也 由原来的绿色变为黄色, Zhao 等 ${ }^{47}$ 也合成了主配体 含有三芳基硼的重金属铱配合物 $\mathbf{2 3}$, 加入 $\mathrm{F}^{-}$后, 铱 配合物的发射被完全淬灭, 得到了一种对于 $\mathrm{F}^{-}$的高 选择、高灵敏的“turn-off”型传感器. 之后, 他们对该 工作做了更深入的研究, ${ }^{48,49}$ 合成了含有咔唑单元的 两种寊聚物 (主链和侧链型) 作为能量的给体, 与配 体含有三苯基嗍的二氯桥反应, 得到了两种离子型 铱配合物 $24 、 25$, 并研究了这两种化合物对 $\mathrm{F}^{-}$的响 


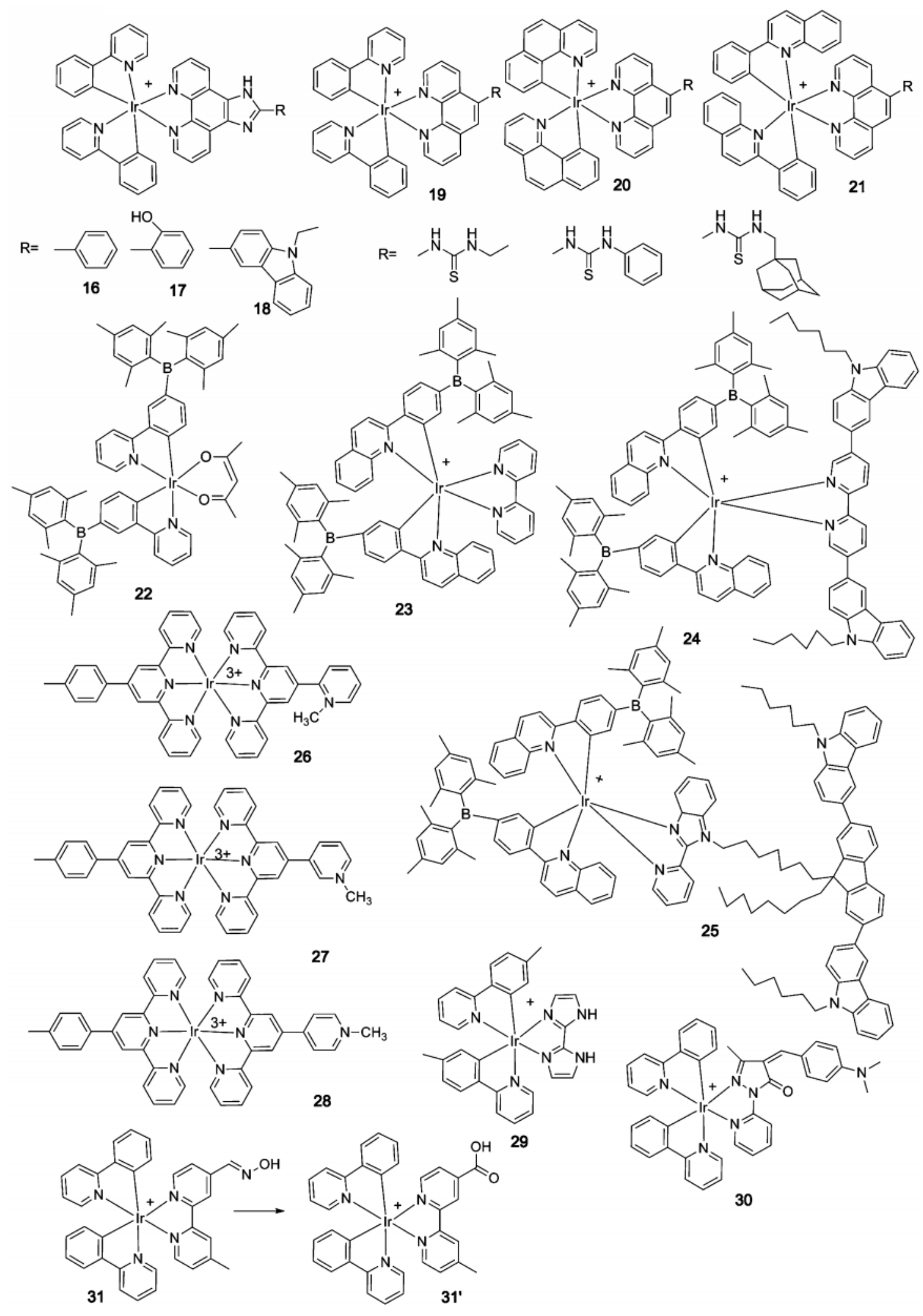

图 2 铱配合物用于阴离子传感器

Fig.2 Application of iridium complexes in chemosensors for anions 
应. 随着 $\mathrm{F}^{-}$的加入, 配合物的能量传递过程发生改 变, 使得铱配合物的特征发射强度逐渐降低, 而辅 助配体的蓝光发射逐渐升高, 相对于侧链型铱配合 物 25, 主链型铱配合物 24 作为比率型传感器的检测 性能更好, 这是由于在主链型铱配合物中, 铱配合 物到咔唑衍生物的能量传递现象更加明显.

Goodall 等 ${ }^{50}$ 比较了三种基于三联吡啶的重金 属铱配合物 26-28 的发光性能, 发现对位异构的配 合物 $\mathbf{2 8}$ 的量子效率低, 且磷光寿命短, 这是由于 $N$ 甲基吡啶上氮与三联吡啶的直接共轭使配体更加 缺电子, 进而使 MLCT 态的能级降低, 当 $\mathrm{Cl}^{-}$加入到 配合物 28 后, $\mathrm{Cl}^{-}$与 $N$-甲基吡啶上的氮作用, 使得配 合物的发光红移而且发射强度降低. Freys 等 ${ }^{51}$ 合成 了辅助配体为联咪唑的重金属铱配合物 29, 该配合 物能与苯甲酸衍生物 1:1 络合, 生成含有苯甲酸的 盐, 使得溶液的发光逐渐淬灭, 这是由于苯甲酸的 加入使得 MLCT 态淬灭.

Lou 等 ${ }^{2}$ 合成了离子型铱配合物 $\mathbf{3 0}$, 比较了配 合物与该配合物的辅助配体作为 $\mathrm{CN}^{-}$的传感器的性 能, 当 $\mathrm{CN}^{-}$加入后配合物 $\mathbf{3 0}$ 的颜色由粉红变为无 色, 而且溶液的苂光发射在 $520 \mathrm{~nm}$ 处有新峰产生, 同时, 配合物的电化学性能也发生了改变, 相比于 配合物的辅助配体, 配合物 $\mathbf{3 0}$ 的苂光变化更加明 显, 而且响应时间更加短.

次氯酸根是由双氧水和氯气产生的, 它具有杀 菌功能, 但是过量的 $\mathrm{ClO}^{-}$会对生物体有危害, 可能 会引起癌症和动脉硬化等疾病. Zhao 等 ${ }^{53}$ 合成了辅 助配体含有醛肪 $(-\mathrm{C}=\mathrm{N}-\mathrm{OH})$ 基团的离子型铱配 合物 31, 当加入 $\mathrm{ClO}^{-}$后, 体系的发射明显增强, 配合 物 31'生成, 该传感器抗干扰性能良好, 而且配合物 31'对 $\mathrm{pH}$ 值响应良好, 随着体系 $\mathrm{pH}$ 值的升高, 配合 物的羧基逐渐被质子化使得配合物的发射增强.

目前重金属铱配合物已广泛应用于阴离子传 感器, 但是相比于阳离子传感器, 基于重金属铱配 合物的阴离子传感器能检测的离子种类还是较少. 而且目前大部分铱配合物对阴离子的选择性不高, 这要求我们根据这些阴离子的特性研究出选择性 和灵敏度更高的阴离子传感器.

\section{3 氨基酸传感器}

在生物体内氨基酸扮演着非常重要的角色, 某 些氨基酸的过多或者缺失都会引起疾病, 比如人体 内如果半胱氨酸含量不正常, 会引起皮肤病变和肝 功能混乱, 而高半胱氨酸是引起老年痴呆症和心血
管疾病的原因之一, 54,55 因此, 在生命科学研究领域, 对这些氨基酸的定性及定量检测非常重要(如图 3 所示).

在发光基团上引入甲酰基, 利用甲酰基与半胱 氨酸和高半胱氨酸的闭环反应可以得到对这两种 氨基酸的传感器. ${ }^{56,57} 2007$ 年 Chen 等 ${ }^{58}$ 合成了主配体 含有甲酰基的中性铱配合物 32, 通过对滴定前后配 合物的核磁测试发现加入半胱氨酸和高半胱氨酸 后, 甲酰基与它们发生了闭环反应, 高半胱氨酸加 入后, 铱配合物的发射发生了蓝移, 而且发射强度 明显增强, 但是半胱氨酸的加入却使得荧光淬灭, 通过理论计算发现, 这是由于半胱氨酸的加入使得 配合物的 MLCT 激发态与生成的噻唑烷之间发生 了电荷转移. 2010 年, 他们 ${ }^{59}$ 将联吡啶作为辅助配 体, 得到离子型铱配合物 $\mathbf{3 3}$, 虽然检测机理类似, 但 是检测结果却完全不同, 该配合物对半胱氨酸和高 半胱氨酸的响应结果类似, 都是红移而且增强, 实 现了对这两种氨基酸的比率识别, 将中性配合物改 为离子型配合物, 使得配合物的水溶性增加, 成功 将检测前后的物质运用到生物标记中.

$\mathrm{Zn}^{2+}$ 与核苷酸有良好的络合能力, Kwon 等 ${ }^{60}$ 借 助锌离子与核苷酸的络合能力得到了一种对核苷 酸有响应的化学传感, 实现了对核苷酸的高灵敏、 高选择的检测, 当往体系中加入核苷酸后, 铱配合 物与咔唑衍生物形成给受体集合体系, 它们之间发 生有效能量传递, 使得体系的发射增强.

双键与硫醇基团在室温下容易发生烯巯加成 反应, 该反应条件温和, 产率高, 一直是检测含硫基 团氨基酸的方法之一。. ${ }^{61,62}$ Zhao 等 ${ }^{63}$ 用羟醛缩合合成 了含双键的联吡啶的衍生物, 作为辅助配体来解离 苯基吡啶的二氯桥后得到铱配合物 $\mathbf{3 4}$, 当半胱氨酸 加入后, 配合物的紫外和苂光都发生了明显的变 化, 配合物的苂光明显增强, 该传感器对高半胱氨 酸也有响应, 但是荧光增强现象没有半胱氨酸明 显, 这是由于配合物的激发态由 ILCT 态变为 LLCT/ MLCT态.

离子型重金属铱配合物 $35^{64}$ 可以作为组氨酸传 感器, 当过量组氨酸加入后, 体系的发光强度增加 了 180 倍, 该物质还可以用于蛋白质的染色, 其染色 效果与 SYPRO Ruby (蛋白质染料) 类似, 而且不需 要退染色步骤和有机溶剂的参与, 染色在 $10 \mathrm{~min}$ 完 成. Liu等 ${ }^{65}$ 也做了类似工作, 他们先合成了辅助配 体含硫的铱配合物 36 , 当过量的 $\mathrm{Hg}^{2+}$ 加入后, 生成 
<smiles></smiles>

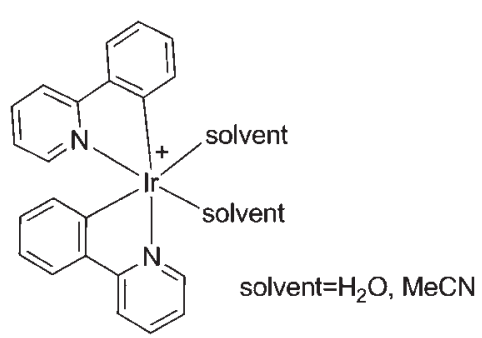

35

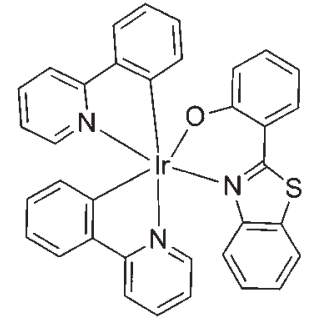

36

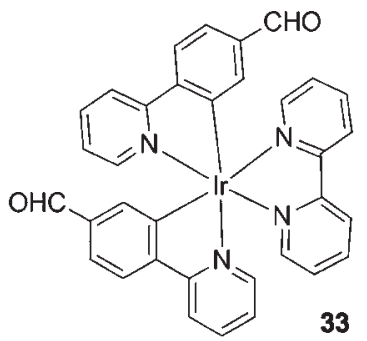<smiles></smiles>

$\mathrm{OHC}$

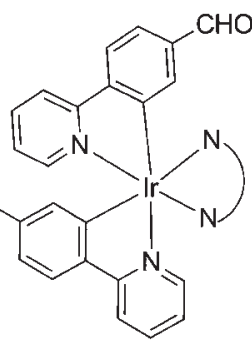<smiles></smiles><smiles>c1cnc2c(c1)ccc1cccnc12</smiles><smiles>Cc1cnc2c(ccc3c(C)c(C)cnc32)c1C</smiles>

图 3 铱配合物用于氨基酸传感器

Fig.3 Application of iridium complexes in chemosensors for amino acids

的物质类似配合物 $\mathbf{3 5}$, 当往里面加入组氨酸后, 发 光明显增强, 利用这个现象, 得到了 “与(AND)”门和 “禁(INHIBIT)”门两种逻辑门。

铱配合物的溶解性能好, 可以通过修饰配体来 提高铱配合物在不同溶剂中的溶解性, 对细胞毒性 低、生物相容性好, 而且铱配合物的稳定性好, 受溶 剂、光、热等的影响小, 利用这些优点, 铱配合物被 广泛运用于生物标记. ${ }^{66-68} \mathrm{Lo}$ 等 ${ }^{69}$ 合成了主配体含有 甲酰基的铱配合物 37-40, 利用甲酰基与生物体中 分子的氨基反应, L-丙氨酸和抗生素蛋白与这些配 合物结合生成了发光的生物共轭物, 这是由于铱配 合物的激发态由 ILCT 态变为 MLCT 态, 最近他们 加大了主配体的共轭度, 合成了一系列铱配合物 41-44, ${ }^{70}$ 将其与牛血清白蛋白反应生成生物共轭 体, 并将这些物质应用到细胞成像中, 发现这类物 质成像稳定, 成像效果明显.

虽然基于铱配合物的化学传感器用于氨基酸 的检测已经发展得很成熟, 但是还是存在缺点, 比 如能检测的氨基酸过于单一, 大多是半胱氨酸和高
半胱氨酸, 以后的研究方向将集中在根据其它氨基 酸的特性设计更多检测其它氨基酸的传感器.

\section{4 氧分子传感器}

氧分子是环境中最常见的气体之一, 氧气在日 常生活及工农业生产中有着极为重要的地位, 氧不 仅与人和动物的生存息息相关, 也与化学、生化反 应以及物理现象关系密切, 因此氧分子的检测非常 重要, 目前, 已经有一些氧分子检测的方法, 比如某 些碳氢聚合物, 但是这些传感器的灵敏度不够高, 容易受外界环境的影响. 金属配合物的磷光会被氧 分子淬灭, 而且淬灭过程中不会产生和消耗氧气, 因此可以被用来作为氧分子传感器. 相比于钓和铂 等重金属配合物, 铱配合物被用于氧分子传感器是 由于其亮度高, 发光寿命长, 斯托克斯能量转移大 而且响应时间短(如图 4所示). 在制备氧分子传感器 的时候, 由于高量子效率的传感器在分散环境中的 灵敏度差, 因此在发展氧分子传感器的过程中允许 灵敏度稍差的检测.

Huynh 等 ${ }^{71}$ 制得了一系列基于铼配合物 $\mathbf{4 5}$, 铱 

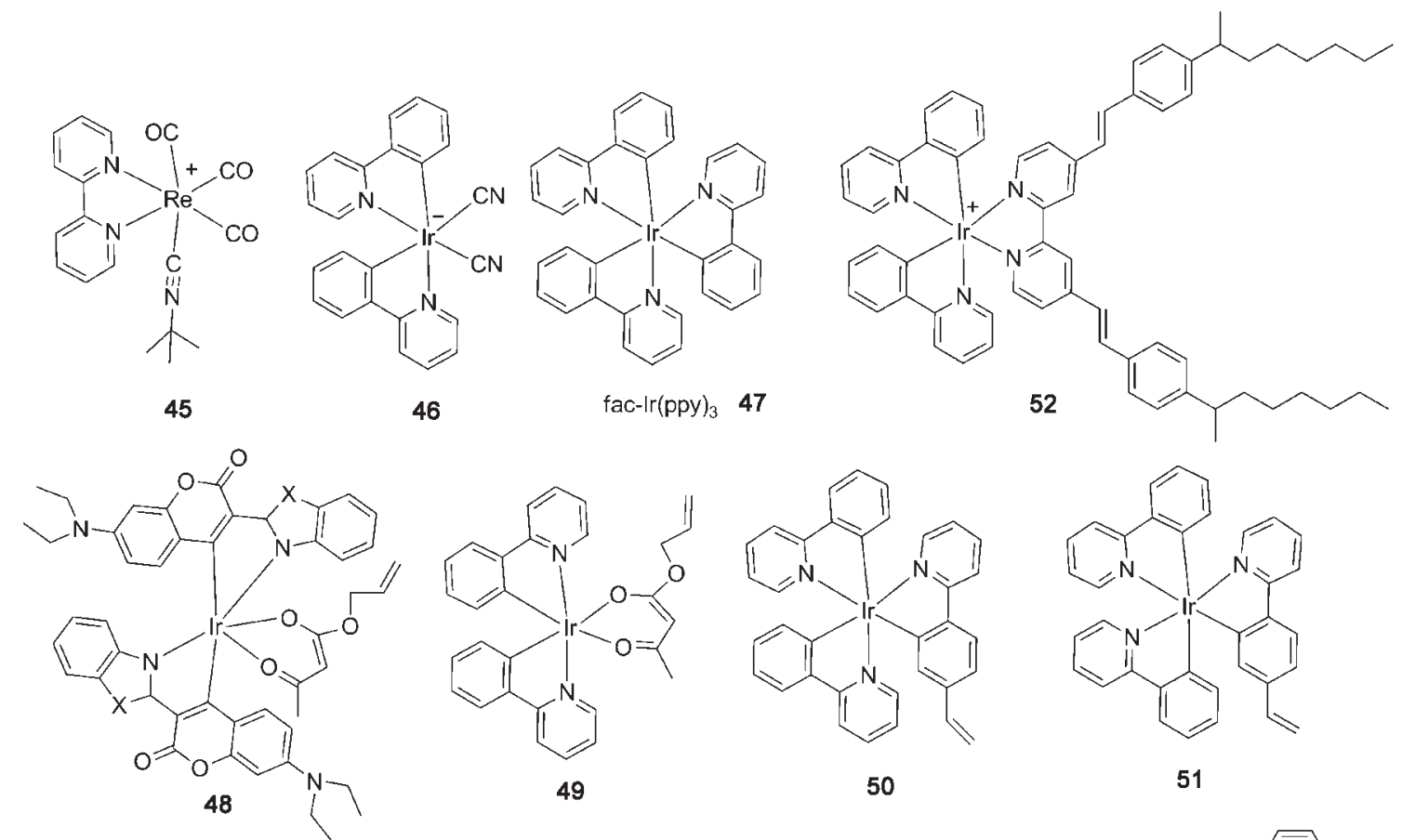

51

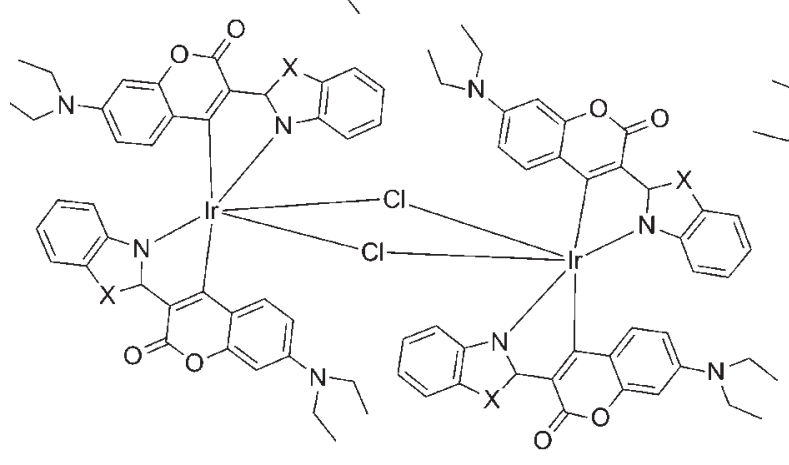

$X=N-M e, O, S \quad 53$

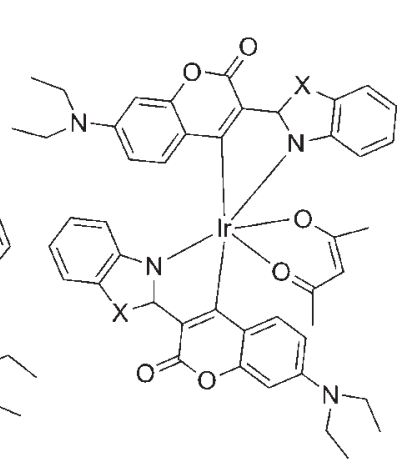

$\mathrm{X}=\mathrm{N}-\mathrm{Me}, \mathrm{O}, \mathrm{S} 5 \mathbf{5 4}$

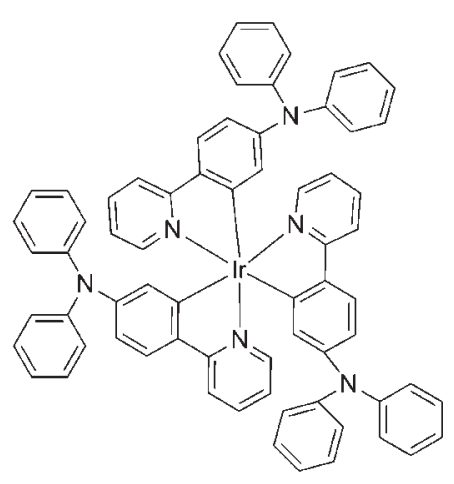

55

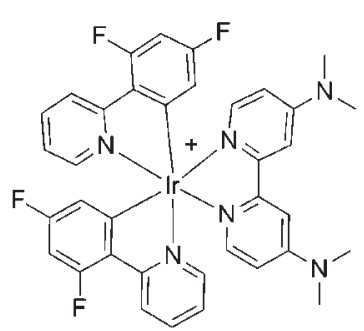

56

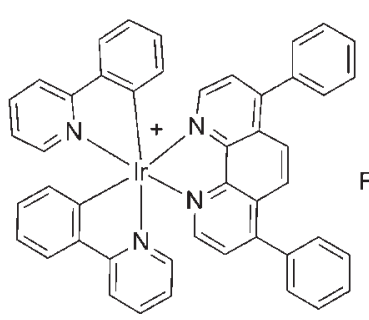

57

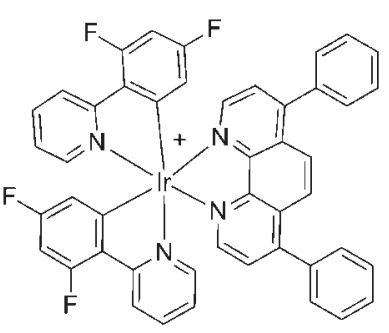

58

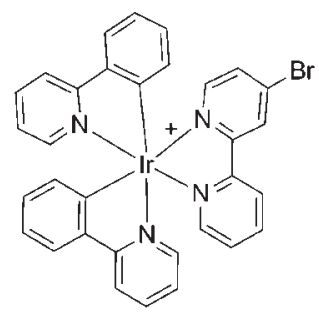

59

图 4 铱配合物用于氧分子传感器

Fig.4 Application of iridium complexes in chemosensors for oxygen molecules

配合物 46 和 47 的氧分子传感器, 在这些传感器中, 基于铱配合物面式-三(2-苯基吡啶)铱(fac- $\left.\operatorname{Ir}(\mathrm{ppy})_{3}\right)$ (47)的传感器展示良好的灵敏度, 它被氧淬灭灵敏 度是八乙基卟吩铂(PtOEP) 的 2.7 倍, 是三(4,7-联 苯-1,10-邻菲啰啉)二氯化钉 $\left(\left[\mathrm{Ru}(\mathrm{dpp})_{3}\right] \mathrm{Cl}_{2}\right)$ 的 5 倍. Derosa 等 ${ }^{72}$ 合成了一些列含双键可聚合的铱配合物 48-51, 并且对其光物理性能和电化学性能进行了
表征, 化合物 48 和 50 拥有较长的苂光寿命和较高 外量子效率, 因此最有希望将这类物质制备成具备 压力传感功能的涂料. 将氧分子传感器应用到生物 领域中, 需要配合物的发光高于 $650 \mathrm{~nm}$, 这是由于 体系中生物分子的发光波长在 550-600 $\mathrm{nm}$ 之间, Madina-Castillo 等 ${ }^{73}$ 设计合成了铱配合物 52, 发光波 长在 $665 \mathrm{~nm}$; 量子效率为 $(0.58 \pm 0.05) \%$, 磷光寿命 
$102 \mu \mathrm{s}$, 将其用于氧分子的检测灵敏度高, 稳定性 好. Borisov等 ${ }^{74}$ 合成了以香豆素为主配体的铱配合 物 53、54, 并且将其应用到氧分子传感器中. 配合物 54 拥有强的发光亮度, 他们将这些配合物固载在聚 苯乙烯薄膜上, 在氧气含量从 $0 \%$ 变化到 $100 \%$ 时, 磷 光逐渐衰减. 相对于其它氧分子传感器, 它不仅灵 敏度高, 而且温度对其影响小, 但是该配合物的光 稳定性不佳, 使得材料只能应用到短期检测中. Mak 等 ${ }^{75}$ 合成了三环铱配合物 $\mathbf{5 5}$, 与 $\operatorname{Ir}(\mathrm{ppy})_{3}$ 相比, 配合 物 55 的发射红移, 由于该配合物的发射寿命长, 量 子效率高而且溶解性能良好, 将其溶解到乙基纤维 素中制得了高灵敏度的氧分子传感器, 使得该材料 可能被应用于光纤传感器和微孔反应板中. Toro 等 ${ }^{76}$ 将一系列离子型铱配合物 56-59 固定到聚苯乙 烯、金属和纳米材料中, 得到一系列氧分子传感器, 研究发现将配合物固载到纳米材料中能提高检测 的灵敏度, 其中, 将配合物 57 固载到 AP200/19 型纳 米材料中得到的传感器展现出最佳的灵敏度, 能够 检测到浓度小于 $10 \%$ 的氧分子.

\section{$2.5 \mathrm{pH}$ 值传感器}

如今, 由于酸碱度在环境、日常生活和人体中 的作用非常重要, 比如有些酶只在某些特定的 $\mathrm{pH}$ 值下才能显示出催化功能, 实时检测 $\mathrm{pH}$ 值已经成
为研究的热点, 到目前为止, 已经有一些对 $\mathrm{pH}$ 值有 响应的有机小分子已经被报道, ${ }^{77-79}$ 相对于有机小分 子, 基于重金属铱配合物的 $\mathrm{pH}$ 传感器具有较长的 磷光寿命, 斯托克斯位移大, 激发和发射容易分辨 等优点, 而且铱配合物有一定的水溶性, 这对检测 体系 $\mathrm{pH}$ 值非常重要(如图 5 所示).

目前检测 $\mathrm{pH}$ 值的化学传感器主要是通过在发 光基团上引入一些可以质子化的官能团, 比如氨 基、羧基、吡啶环等, 这些基团被质子化后, 与发光 基团之间的能量传递发生改变, 进而影响分子的光 化学性能. 基于 $2,2^{\prime}, 6^{\prime}, 2^{\prime \prime}$-三联吡啶的铱配合物的发 光性能很容易受三联吡啶上 $4^{\prime}$ 号位置的官能团的影 响, Licini 等 ${ }^{80}$ 在 $4^{\prime}$ 号位置引入吡啶环, 制得铱配合 物 60, 当体系的酸性增强后, 吡啶环被质子化, 使得 配合物的激发态由 ILCT 态变为 MLCT 态, 当体系 的 $\mathrm{pH}$ 值小于 2 的时候体系的发光寿命和发射强度 都明显降低. 与配合物 60 不同的是, 配合物 61 的 4' 号位置引入了苯酚基团, 该配合物也可以用来作为 $\mathrm{pH}$ 传感器, 当体系的 $\mathrm{pH}$ 值大于 10 时配合物在水溶 液中的颜色由淡黄色变为橘黄色, 而且配合物在 $468 \mathrm{~nm}$ 处有新的吸收带产生. 配合物 $62^{81}$ 质子化前 后的现象与配合物 60 和 61 的类似.

Aoki 等 ${ }^{82}$ 合成了一些列三环金属化铱配合物
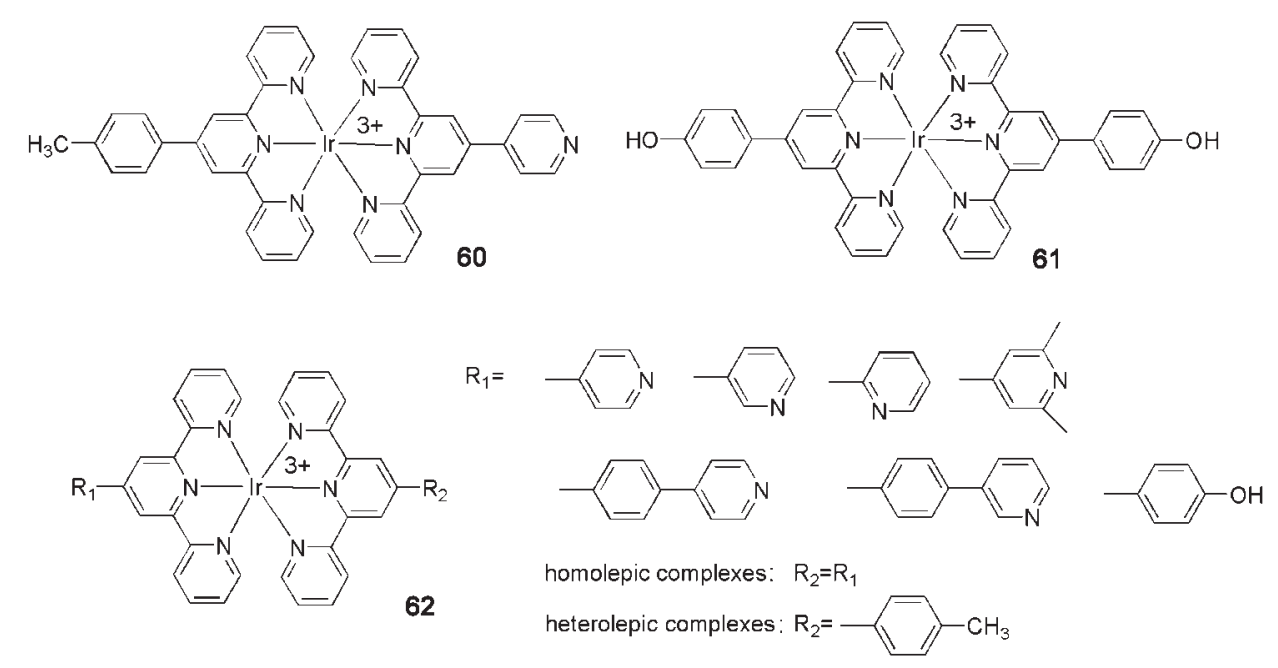<smiles></smiles>

63

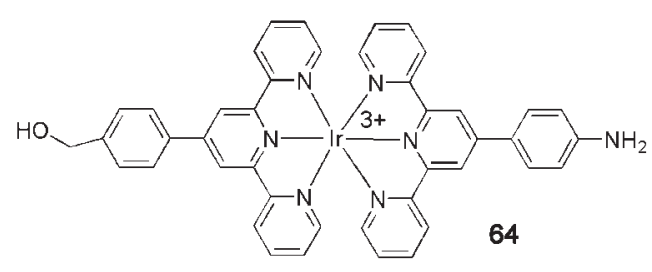

图 5 铱配合物用于 $\mathbf{p H}$ 传感器

Fig.5 Application of iridium complexes in chemosensors for $\mathrm{pH}$

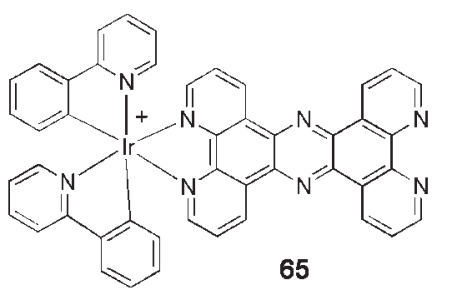

65 
63, 在不同 $\mathrm{pH}$ 值下实现了铱配合物发光颜色从绿 光到红光的改变, 在 $\mathrm{pH}=4$ 时体系的发光在 $531 \mathrm{~nm}$ 处, 而且强度较弱, 随着体系的 $\mathrm{pH}$ 值变大, 发光由 原来的绿光变成红光, 而且发射强度增加. Goldstein 等 ${ }^{83}$ 合成了一系列以三联吡啶为配体的水溶性铱配 合物, 这些配合物在不同溶剂中的吸收光谱不同, 呈现了较强的溶剂效应, 在这些配合物中配合物 64 可用来作为 $\mathrm{pH}$ 传感器, 体系的 $\mathrm{pH}$ 值降低后, 配合物 64 上的氨基开始质子化使得配合物的激发态发生 改变, 配合物的吸收光谱发生变化.

邻菲啰啉经过修饰后可以得到一系列含有喹 喔啉杂环的邻菲啰啉衍生物, 将这类物质作为辅助 配体制得的磷光材料对 $\mathrm{pH}$ 值有很好的响应, 而且 由于该物质可以与 DNA 结合, 可以将其运用到生物 标定中 ${ }^{84,85}$ Chen 等 ${ }^{86}$ 合成了离子型铱配合物 65 , 由 于辅助配体上裸露的氮原子较多, 该配合物的质子 化分三步进行, 使得物质能检测的 $\mathrm{pH}$ 值范围增大, 当 $\mathrm{pH}$ 值从 2 增加到 12 时, 物质的紫外吸收明显降 低.

目前, 基于重金属铱配合物的 $\mathrm{pH}$ 传感器已被 广泛研究, 但是, 还是存在相关的问题, 比如配合物 很难做到完全水溶, 使得测得的 $\mathrm{pH}$ 值不是非常准 确, 而且 $\mathrm{pH}$ 值的检测范围过于狭窄, 发光颜色变化 过于单一, 这加大了市场化的难度.

\section{6 其它传感器}

Liu 等 ${ }^{87}$ 合成了铱配合物 66(如图 6 所示), 发现 其对乙腈蒸气有良好的传感性能, 铱配合物 66 在固 态下以两种形式存在, 这两种形式的颜色分别为红 色和黑色, 当这些固体与乙腈蒸气接触后, 颜色为 黑色的固体变为红色, 而且发射明显增强. 通过单 晶和理论计算分析发现, 当乙腈蒸气加入后, 配合 物的堆积形式发生改变使得配合物在固态下的光 学性能发生明显的变化.

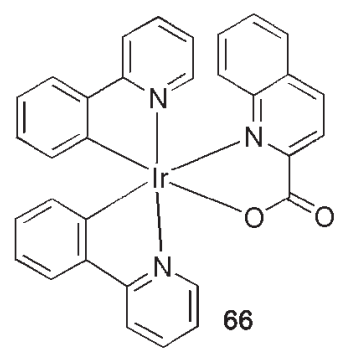

图 6 铱配合物用于其它传感器

Fig.6 Application of iridium complex in chemosensors for others

\section{3 结论与展望}

由于电致发光领域的迅速发展, 铱配合物被广 泛关注, 88,89 研究者对铱配合物的结构、电学性能、光 学性能等进行了细致的研究, 并将其运用到其它领 域, 比如: 光催化储氢、 ${ }^{90,91}$ 太阳能电池、 ${ }^{92}$ 电化学电 池、年-96 化学传感器、电化学发光分析 ${ }^{97}$ 等. 基于铱配 合物的化学传感器发展势头迅猛, 但是还是存在相 关问题需要改进. (1) 目前铱配合物的发光性能容易 受其它环境因素的影响, 比如氧气, 在空气中, 铱配 合物的发射强度会随着氧气含量的增加而减弱, 这 使得传感器的灵敏度降低而且检测时需要考虑空 气中氧气含量, 检测变得麻烦, 因此, 合成一些光学 性能受环境影响小的铱配合物尤为重要. (2) 相比于 基于纯有机分子的荧光传感器, 基于铱配合物的磷 光传感器发展还是不够成熟, 能检测的物质较少, 这是由于基于铱配合物的磷光传感器的设计受铱 配合物的合成方法和铱配合物的能量传递方式的 限制. 这要求我们对铱配合物的合成方法进行改 良. (3) 要将铱配合物的传感器运用到生物体内, 由 于其它溶剂对细胞的毒性强, 因此需要铱配合物拥 有良好的水溶性, 以后对水溶性铱配合物的研究非 常重要, 另一方面, 紫外线对细胞的伤害也非常大, 目前大多数的铱配合物的发射都是靠紫外光激发, 因此需要尽量将铱配合物的最佳激发波长红移, 比 如制备发射波长在近红外区的磷光化学传感器.

\section{References}

(1) Demas, J. N.; DeGraff, B. A. Coord. Chem. Rev. 2001, 211, 317.

(2) Cheng, Y. W.; Yang, Z.; Wei, H.; Wang, Y. Y.; Wei, L. M.; Zhang, Y. F. Acta Phys. -Chim. Sin. 2010, 26, 3127. [程应武, 杨 志, 魏 浩, 王艳艳, 魏良明, 张亚非. 物理化学学报, 2010, 26, 3127.]

(3) Li, M. J.; Chu, B. W. K.; Zhu, N. Y.; Yam, V. W. W. Inorg. Chem. 2007, 46, 720.

(4) Amoroso, A. J.; Arthur, R. J.; Coogan, M. P.; Court, J. B.; Moreira, V. F.; Hayes, A. J.; Lloyd, D.; Millet, C.; Pope, S. J. A. New J. Chem. 2008, 7, 1097.

(5) Carraway, E. R.; Demas, J. N.; DeGraff, B. A.; Baeon, J. R. Anal. Chem. 1991, 63, 337.

(6) Ge, F.; Cao, R. G.; Zhu, B.; Li, J. J.; Xu, D. S. Acta Phys. -Chim. Sin. 2010, 26, 1779. [戈 芳, 曹瑞国, 朱斌, 李经建, 徐东升. 物理化学学报, 2010, 26, 1779.]

(7) Lin, Z. H.; Zhao, Y. G.; Duan, C. Y.; Zhang, B. G.; Bai, Z. P. Dalton Trans. 2006, 30, 3678.

(8) Lo, H. S.; Yip, S. K.; Wong, K. M. C.; Zhu, N. Y.; Yam, V. W. W. Organometallics 2006, 25, 3537. 
(9) McFarland, S. A.; Finney, N. S. Chem. Commun. 2003, No. 3, 388.

(10) Yu, X. H.; Ge, G. P.; Zhang, G. L.; Guo, H. Q. Acta Phys. -Chim. Sin. 2010, 26, 1184. [于晓航, 葛国平, 张国林, 郭海清. 物理化学学报, 2010, 26, 1184.]

(11) Tong, B.; Mei, Q.; Wang, S.; Fang, Y.; Meng, Y.; Wang, B. J. Mater. Chem. 2008, 18, 1636.

(12) Kirgan, R. A.; Sullivan, B. P.; Rillema, D. P. Top. Curr. Chem. 2007, 281, 45 .

(13) Roundhill, D. M. Photochemistry and Photophysics of Metal Complexes; Plenum Press: New York, 1994.

(14) Kumar, A.; Sun, S. S.; Lees, A. J. Coord. Chem. Rev. 2008, 252, 922.

(15) Cannizzo, A.; Blanco-Rodriguez, A. M.; Nahhas, A. E.; Sebera, J.; Zalis, S.; Chergui, M. J. Am. Chem. Soc. 2008, 130, 8967.

(16) King, K. A.; Watts, R. J. J. Am. Chem. Soc. 1987, 109, 1589.

(17) Ayala, N. P.; Flynn, C. M., Jr.; Sacksteder, L.; Demas, J. N.; DeGraff, B. A. J. Am. Chem. Soc. 1990, 112, 3837.

(18) Polson, M.; Fracasso, S.; Bertolasi, V.; Ravaglia, M.; Scandola, F. Inorg. Chem. 2004, 43, 1950.

(19) Xia, W. S.; Schmehl, R. H.; Li, C. J. J. Am. Chem. Soc. 1999, 121,5599 .

(20) Valeur, B.; Leray, I. Coord. Chem. Rev. 2000, 205, 3.

(21) Silva, A. P.; Fox, D. B.; Huxley, A. J. M.; Moody, T. S. Coord Chem. Rev. 2000, 205, 41.

(22) Frausto da Silva, J. J. R.; Williams, R. J. P. The Biological Chemistry of the Elements: the Inorganic Chemistry of Life; Clarendon: Oxford, 1991.

(23) Gutknecht, J. J. Membr. Biol. 1981, 61, 61.

(24) Ho, M. L.; Cheng, Y. M.; Wu, L. C.; Chou, P. T.; Lee, G. H.; Hsu, F. C.; Chi, Y. Polyhedron 2007, 26, 4886.

(25) Chen, X. Y.; Shi, J.; Li, Y. M.; Wang, F. L.; Wu, X.; Guo, Q. X.; Liu, L. Org. Lett. 2009, 11, 4426.

(26) Xu, Z.; Baek, K. H.; Kim, H. N.; Cui, J.; Qian, X.; Spring, D. R.; Shin, I.; Yoon, J. J. Am. Chem Soc. 2010, 132, 601.

(27) Xue, L.; Liu, C.; Jiang, H. Org. Lett. 2009, 11, 1655.

(28) Araya, J. C.; Gajardo, J.; Moya, S. A.; Aguirre, P.; Toupet, L.; Williams, J. A. G.; Escadeillas, M.; LeBozec H.; Guerchais, V. New J. Chem. 2010, 34, 21.

(29) Zhao, N.; Wu, Y. H.; Wen, H. M.; Zhang, X.; Chen, Z. N. Organometallics 2009, 28, 5603.

(30) Charbonniere, L. J.; Ziessel, R. F.; Sans, C. A.; Harriman, A. Inorg. Chem. 2003, 42, 3466.

(31) Li, C. K.; Lu, X. X.; Wong, K. M. C.; Chan, C. L.; Zhu, N.; Yam, V. W. W. Inorg. Chem. 2004, 43, 7421.

(32) Muegge, B. D.; Richter, M. M. Anal. Chem. 2002, 74, 547.

(33) Schmittel, M.; Lin, H. Inorg. Chem. 2007, 46, 9139.

(34) Lin, H.; Cinar, M. E.; Schmittel, M. Dalton Trans. 2010, 39, 5130 .

(35) Ho, M. L.; Hwang, F. M.; Chen, P. N.; Hu, Y. H.; Cheng, Y. M.; Chen, K. S.; Lee, G. H.; Chi, Y.; Chou, P. T. Org. Biomol.
Chem. 2006, 4, 98.

(36) Brandel, J.; Sairenji, M.; Ichikawa, K.; Nabeshima, T. Chem. Commun. 2010, 46, 3958.

(37) Zhao, Q.; Cao, T. Y.; Li, F. Y.; Li, X. H.; Jing, H.; Yi, T.; Huang, C. H. Organometallics 2007, 26, 2077.

(38) Zhao, Q.; Liu, S. J.; Li, F. Y.; Yi, T.; Huang, C. H. Dalton Trans. 2008, 29, 3836 .

(39) Shi, H. F.; Liu, S. J.; Sun, H. B.; Xu, W. J.; Zhao, Q.; Huang, W. Chem. Eur. J. 2010, 16, 12158.

(40) Yang, H.; Qian, J. J.; Li, Z. G.; Li, D. R.; Wu, H. X.; Yang, S. P. Inorg. Chim. Acta 2010, 263, 1755.

(41) Beer, P. D.; Gale, P. A. Angew. Chem. Int. Edit. 2001, 40, 486.

(42) Special Issues on Anion Receptors, Coord. Chem. Rev. 2003, $240(1-2)$.

(43) Martnez-Mnez, R.; Sancenon, F. Chem. Rev. 2003, 103, 4419.

(44) Zhao, Q.; Liu, S. J.; Shi, M.; Li, F. Y.; Jing, H.; Yi, T.; Huang, C. H. Organometallics 2007, 26, 5922.

(45) Lo, K. K. W.; Lau, J. S. Y.; Lo, D. K. K.; Lo, L. T. L. Eur. J. Inorg. Chem. 2006, 4054.

(46) You, Y.; Park, S. Y. Adv. Mater. 2008, 20, 3820.

(47) Zhao, Q.; Li, F.; Liu, S.; Yu, M.; Liu, Z.; Yi, T.; Huang, C. Inorg. Chem. 2008, 47, 9256.

(48) Xu, W.; Liu, S.; Zhao, X.; Sun, S.; Cheng, S.; Ma, T.; Sun, H.; Zhao, Q.; Huang, W. Chem. Eur. J. 2010, 16, 7125.

(49) Xu, W.; Liu, S.; Sun, H.; Zhao, X.; Zhao, Q.; Sun, S.; Cheng, S.; Ma, T.; Zhou, L.; Huang, W. J. Mater. Chem. 2011, 21, 7572.

(50) Goodall, W.; Williams, J. A. G. J. Chem. Soc. Dalton Trans. 2000, 17, 2893.

(51) Freys, J. C.; Bernardinelli, G.; Wenger, O. S. Chem. Commun. 2008, No. 36, 4267.

(52) Lou, B.; Chen, Z. Q.; Bian, Z. Q.; Huang, C. H. New J. Chem. 2010, 34, 132.

(53) Zhao, N.; Wu, Y. H.; Wang, R. M.; Shi, L. X.; Chen, Z. N. Analyst 2011, 136, 2277.

(54) Shahrokhian, S. Anal. Chem. 2001, 73, 5972.

(55) Seshadri, S.; Beiser, A.; Selhub, J.; Jacques, P. F.; Rosenberg, I. H.; D'Agostino, R. B.; Wilson, P. W. F. N. Engl. J. Med. 2002, 346,476

(56) Lin, W.; Long, L.; Yuan, L.; Cao, Z.; Chen, B.; Tan, W. Org. Lett. 2008, 10, 5577.

(57) Zhang, X.; Ren, X.; Xu, Q.; Loh, K. P.; Chen, Z. K. Org. Lett. 2008, 10, 1257.

(58) Chen, H. L.; Zhao, Q.; Wu, Y. B.; Li, F. Y.; Yang, H.; Yi, T.; Huang, C. H. Inorg. Chem. 2007, 46, 11075.

(59) Zhao, Q.; Xiong, L.; Chen, H.; Wu, Y.; Dong, Z.; Zhou, Z.; Li, F. Inorg. Chem. 2010, 49, 6402.

(60) Kwon, T. H.; Kim, H. J.; Hong, J. I. Chem. Eur. J. 2008, 14, 9613.

(61) Matsumoto, T.; Urano, Y.; Shoda, T.; Kojima, H.; Nagano, T. Org. Lett. 2007, 9, 3375.

(62) Huo, F. J.; Sun, Y. Q.; Su, J.; Chao, J. B.; Zhi, H. J.; Yin, C. X. 
Org. Lett. 2009, 11, 4918.

(63) Zhao, N.; Wu, Y. H.; Shi, L. X.; Lin, O. P.; Chen, Z. N. Dalton Trans. 2010, 39, 8288.

(64) Ma, D. L.; Wong, W. L.; Chung, W. H.; Chan, F. Y.; So, P. K.; Lai, T. S.; Zhou, Z. Y.; Leung, Y. C.; Wong, K. Y. Angew. Chem Int. Edit. 2008, 47, 3735.

(65) Liu, Y.; Li, M.; Zhao, Q.; Wu, H.; Huang, K.; Li, F. Inorg. Chem. doi: 10.1021/ic102481x.

(66) Zhao, Q.; Yu, M.; Shi, L.; Liu, S.; Li, C.; Shi, M.; Zhou, Z.; Huang, C.; Li, F. Organometallics 2010, 29, 1086.

(67) Leung, S. K.; Kwok, K. Y.; Zhang, K. Y.; Lo, K. K. W. Inorg. Chem. 2010, 49, 4984.

(68) Zhang, K. Y.; Lo, K. K. W. Inorg. Chem. 2009, 48, 6011.

(69) Lo, K. K. W.; Chung, C. K.; Zhu, N. Chem. Eur. J. 2003, 9, 475.

(70) Lee, P. K.; Liu, H. W.; Louie, M. W.; Yiu, S.; M.; Lo, K. K.W. Dalton Trans. 2010, 40, 2180.

(71) Huynh, L.; Wang, Z.; Yang, J.; Stoeva, V.; Lough, A.; Manners, I.; Winnik, M. A. Chem. Mater. 2005, 17, 4765.

(72) Derosa, M. C.; Hodgson, D. J.; Enright, G. D.; Dawson, B.; Evans, C. E. B.; Crutchley, R. J. J. Am. Chem. Soc. 2004, 126, 7619.

(73) Madina-Castillo, A. L.; Fernandez-Sanchez, J. F.; Klein, C.; Nazeeruddin, M. K.; Segura-Carretero, A.; FernandezGutierrez, A.; Grätzel, M.; Spichiger-Keller, U. E. Analyst 2007, 132,929 .

(74) Borisov, M. S.; Klimant, I. Anal. Chem. 2007, 79, 7051.

(75) Mak, C. S. K.; Pentlehner, D.; Stich, M.; Wolfbeis, O. S.; Chan, W. K.; Yersin, H. Chem. Mater. 2009, 21, 2173.

(76) Toro, M. M.; Fernandez-Sanchez, J. F.; Baranoff, E.; Nazeeruddin, M. K.; Grätzel, M.; Fernandez-Gutierrez, A. Talanta 2010, 82, 620 .

(77) Ohmichi, T.; Kawamoto, Y.; Wu, P.; Miyoshi, D.; Karimata, H.; Sugimoto, N. Biochemistry 2005, 44, 7125.

(78) Whitaker, J. E.; Haughland, R. P.; Prendergast, F. G. Anal. Biochem. 1991, 194, 330.

(79) Lobnik, A.; Oehme, I.; Murkovic, I.; Wolfbeis, O. S. Anal. Chim. Acta 1998, 376, 159.
(80) Licini, M.; Williams, J. A. G. Chem. Commun. 1999, No. 19, 1943.

(81) Arm, K. J.; Leslie, W.; Williams, J. A. G. Inorg. Chim. Acta 2006, 359, 1222.

(82) Aoki, S.; Matsuo, Y.; Ogura, S.; Ohwada, H.; Hisamatsu, Y.; Moromizato, S.; Shiro, M.; Kitamura, M. Inorg. Chem. 2011, 50,806 .

(83) Goldstein, D. C.; Cheng, Y. Y.; Schmidt, T. W.; Bhandbhade, M.; Thordarson, P. Dalton Trans. 2011, 40, 2053.

(84) Han, M.; Chen, Y. M.; Wang, K. Z. New J. Chem. 2008, 32, 970.

(85) Gill, M. R.; Garcia-Lara, G.; Foster, S. J.; Smythe, C.; Battaglia, G.; Thomas, L. A. Nature Chemistry 2009, 1, 662.

(86) Chen, Y.; Zhang, A.; Liu, Y.; Wang, K. Journal of Organometallic Chemistry 2011, 696, 1716.

(87) Liu, Z.; Bian, Z.; Bian, J.; Li, Z.; Nie, D.; Huang, C. Inorg. Chem. 2008, 47, 8025 .

(88) Xiao, L.; Chen, Z.; Qu, B.; Luo, J.; Kong, S.; Gong, Q.; Kido, J. Adv. Mater. 2011, 23, 926.

(89) Tang, X. Q.; Yu, J. S.; Li, L.; Wang, J.; Jiang, Y. D. Acta Phys. -Chim. Sin. 2008, 24, 1012. [唐晓庆, 于军胜, 李 璐, 王 军, 蒋亚东. 物理化学学报, 2008, 24, 1012.]

(90) Tinker, L. L.; McDaniel, N. D.; Curtin, P. N. Chem. Eur. J. 2007, 13, 8723.

(91) McDaniel, N. D.; Coughlin, F. J.; Tinker, L. L. J. Am. Chem. Soc. 2008, 130, 210.

(92) Nastasi, F.; Puntriero, F.; Campagna, S. Chem. Commun. 2007, No. 34, 3556.

(93) He, L.; Duan, L.; Qiao, J.; Dong, G.; Wang, L.; Qiu, Y. Chem. Mater. 2010, 22, 3535.

(94) Mydlad, M.; Bizzarri, C.; Hartmann, D.; Sarfert, W.; Schmid, G.; Cola, D. L. Adv. Funct. Mater. 2010, 20, 1812.

(95) Lowry, M. S.; Bernhard, S. Chem. Eur. J. 2006, 12, 7970.

(96) He, L.; Duan, L.; Qiao, J.; Wang, R.; Wei, P.; Wang, L.; Qiu, Y. Adv. Funct. Mater. 2008, 18, 2123.

(97) Zapata, F.; Caballero, A.; Espionsa, A.; Tarraga, A.; Moina, P. Dalton Trans. 2009, 20, 3900. 